Can a brief interaction with online, digital art improve wellbeing?:

A comparative study of the impact of online art and culture presentations on mood, stateanxiety, subjective wellbeing, and loneliness

MacKenzie D. Trupp ${ }^{* 1}$, Giacomo Bignardi ${ }^{2}$, Kirren Chana ${ }^{1}$, Eva Specker ${ }^{1}$, Matthew Pelowski ${ }^{1,3}$

${ }^{1}$ Department of Cognition, Emotion, and Methods in Psychology, Faculty of Psychology, Universität Wien, Vienna, Austria

${ }^{2}$ Max Planck School of Cognition, Leipzig, Germany

${ }^{3}$ Vienna Cognitive Science Hub, University of Vienna, Vienna, Austria

Address for correspondence:

MacKenzie D. Trupp, Department of Cognition, Emotion, and Methods in Psychology, Faculty of Psychology, Universität Wien, Vienna, Austria. Wächtergasse 1/301, 1010 Vienna, Austria, (mackenzietrupp@gmail.com), (D) https://orcid.org/0000-0003-3542-7787,

Data availability: The data and analysis scripts supporting the results of this paper are openly available at https://github.com/giacomobignardi/Trupp-online-art-wellbeing.

Draft version: 1.0, 02.07.2021. This paper has not been peer reviewed. Please do not copy without author's permission. License CC BY 4.0.

Keywords: Cultural Engagement; Receptive Art Engagement; Wellbeing; Mental health; Digital Art 


\title{
Can a brief interaction with online, digital art improve wellbeing?: \\ A comparative study of the impact of online art and culture presentations on mood, state- anxiety, subjective wellbeing, and loneliness
}

\begin{abstract}
:
When experienced in-person, engagement with art has been associated - in a growing body of evidence - with positive outcomes in wellbeing and mental health. This represents an exciting new field for psychology, curation, and health interventions, suggesting a widelyaccessible, cost-effective, and non-pharmaceutical means of regulating factors such as mood or anxiety. However, can similar impacts be found with online presentations? If so, this would open up positive outcomes to an even-wider population - a trend becoming accelerated due to the current Covid-19 pandemic. Despite its promise, this question, and the underlying mechanisms of art interventions and impacts, has largely not been explored. Participants $(\mathrm{N}=84)$ were asked to engage one of two online exhibitions from Google Arts and Culture (a Monet painting or a similarly-formatted display of Japanese culinary traditions). With just 1-2 minutes' exposure, both improved negative mood, state-anxiety, loneliness, and wellbeing. Stepdown analysis suggested the changes can be explained primarily via negative mood, while improvements in mood correlated with aesthetic appraisals and cognitive-emotional experience of the exhibition. However, no difference was found between exhibitions. We discuss the findings in terms of applications and targets for future research.
\end{abstract}

Keywords: Cultural Engagement; Receptive Art Engagement; Wellbeing; Mental health; Digital Art 


\section{Can a brief interaction with online, digital art improve wellbeing?: \\ A comparative study of the impact of online art and culture presentations on mood, state- anxiety, subjective wellbeing, and loneliness}

With the spread of the novel Coronavirus around early 2020, governments asked their citizens to stay at home to slow the rate of infection and protect the most vulnerable, forcing large parts of society to close their doors and everyday activities to move online. This included engagement with art and cultural institutions, which greatly increased online access to collections to connect with people now stuck at home (Radermecker, 2020; Samaroudi et al., 2020). Virtual tours emerged, encouraging people to 'visit the museum from their couches' (Romano, 2020), and for the first time, engaging with art and culture online was on the minds of a broad audience (Unitt, 2020).

At the same time, many online formats of art and culture signalled their potential application for a pragmatic purpose - ameliorating the negative pandemic-related effects on wellbeing and mental health. A number of projects and discussion in the press were built around suggestions that a brief engagement with a few works of art, or a trip to a virtual museum, might help sooth anxiety, boost one's mood (Barbican Center, 2020), provide better wellbeing (International Arts and Mind Lab, 2020), or even help those isolated and lonely (Belvedere Museum, 2020). These aims seem to have been well received by the public, especially by those with low wellbeing and mental health issues, with evidence suggesting that a notable portion of individuals were, for the first time, seeking out online art encounters (Bu et al., 2021).

These developments are not unique. Rather, the present world-wide pandemic accelerated two trends that have come to define the arts' pragmatic application for wellbeing and health: (1) 
Researchers and practitioners have considered art as a powerful tool to positively modulate wellbeing, which is widely-accessible, cost-effective, and non-pharmaceutical. In a growing body of evidence, when experienced in-person, engagements with art have been associated with positive outcomes (Fancourt \& Finn, 2019 for review). (2) With the ubiquity of the internet, it is being increasingly recognized that digital, online formats might reach an even wider audience and present new possibilities for health-related use. If online engagement could provide a systematic impact, this would represent an even more cost-effective means of reaching large numbers in society (Clayton \& Potter, 2017; Thomson et al., 2018), especially individuals who cannot visit museums, bringing doses of art into homes, hospital beds, or places of work without installation of a costly artwork. As put by one museum director (Gilman, in Rice, 2020), summarizing these dual trends changing the role of the arts, "right now, art... [is] more important than ever. It is one of the key things sustaining us while we are cocooning at home," especially in its new online presence, “...it is what will nourish us as we adjust to the new normal of a post-COVID-19 world."

However, despite this promise, this also raises several questions that have not seen much empirical research: Can art or cultural engagements actually impact mental states such as wellbeing, state-anxiety, mood, satisfaction with life, or loneliness? If so, in which ways and by how much? An answer to these questions has immediate pragmatic importance and raises important issues about the role and nature of typical art interventions, the potential importance of 'real' artworks, and which factors might be key when considering impacts on wellbeing. Similarly, especially considering the wealth of other interventions that might be found onlinehow does online art stack up against similar, non-art cultural engagements, such as other materials that might typically be found in museums and are similarly moving to the digital 
realm? Is there anything particularly different about engaging with visual art? Further, this topic raises several tangential questions of interest, regarding how the nature of the art experience, appraisal, dosage, and duration of an online exhibition might maximize or unlock specific wellbeing outcomes.

This is the aim of the present paper. Employing a quasi-randomized design in which individuals, via the internet, engaged briefly with art content (impressionist painting), we explore the potential impact on a number of factors - wellbeing (mood, life satisfaction, and subjective wellbeing), state anxiety, and loneliness - measured before and after viewing. We compare this intervention to another type of online cultural content (a display of Japanese culinary traditions), which employed a similar presentation format, but was generally expected to be seen as 'not art' by participants and aligning more with receptive engagement such as visiting a history or science museum. We also consider secondary topics in an exploratory analysis, such as the nature of the experience at the appraisal and cognitive-emotional level, viewing time, individuals' label of the stimuli as 'art,' and the interrelation of dependent wellbeing variables.

\section{Background-(Online) Art as a Wellbeing Intervention}

The argument that art, in an online format, could be a tool for addressing wellbeing and mental health can be traced through past research; as can many outstanding questions.

Engagement with a variety of forms of arts and culture - in person - is now welldocumented to support wellbeing and mental health. A 2019 World Health Organization review, included, for example, results from more than 900 publications (Fancourt \& Finn, 2019) suggesting that engaging with various artistic or cultural activities can lead to meaningful impacts both in regards to preventative capacities (maintaining physical and mental wellbeing) and in support of a wide range of psychological issues. Among the many possible receptive or 
creative interventions (attending performances, making or viewing visual art or music, etc), visually engaging artwork or visiting museums and galleries have increasingly been employed in partnership with public health initiatives, and healthcare providers in several countries have started to prescribe such activities as psychological health interventions (Camic \& Chatterjee, 2013; Chatterjee \& Noble, 2017; Packer \& Bond, 2010; Thomson et al., 2018; Todd et al., 2017). Among the various effects, empirical evidence has shown that on-site art interactions have been particularly associated with decreased loneliness (Todd et al., 2017; Tymoszuk et al., 2019, 2020), improved mental health (symptoms of anxiety and depression; Clayton \& Potter, 2017; Hansen et al., 2015; Roberts et al., 2011), and increased mood and subjective wellbeing (Bennington et al., 2016; Binnie, 2010; Davies et al., 2016; Hansen et al., 2015; Ho et al., 2015; Karnik et al., 2014; Roberts et al., 2011; Wang et al., 2020). For example, similar to the interventions that will be explored in this paper, Clow and Fredhoi (2006) asked individuals to take a 35-minute visit to an art gallery on their lunch break and found that even short exposures lead to significantly lower self-reported stress ( $\sim 2.4$ points on a pre-/post-visit 10 -point scale) and cortisol concentrations (see Binnie, 2010; Ho et al., 2015 for similar examples using pre-post assessments).

Impacts have also been found in paradigms eliminating the activity of visiting a museum. For example, Karnik et al. (2014) showed that simply hanging paintings of restorative nature scenes in common rooms led psychiatric patients to have reduced agitation (as reported by nurses) and need for anxiety medication compared to days without the paintings. Similar studies of art in patient or common rooms have been found to improve the anxiety, stress levels, depression, mood, and general wellbeing of both patients and staff (Ho et al., 2015; Nanda et al., 
2011; Staricoff et al., 2003; Ulrich \& Gilpin, 2003). See also Kweon et al. (2008) for similar paradigms showing lower reported stress following art installations in an office.

The explanations of such impacts are themselves under debate (Mastandrea et al., 2019). However, they have been suggested to involve enjoyable or pleasurable experiences (Chatterjee \& Noble, 2017; Fancourt et al., 2020; Sachs et al., 2015), which might improve aspects of subjective wellbeing by regulating mood. They may be tied to shared social, communal factors (Cuypers et al., 2012; Roberts et al., 2011), escapism or removal from daily routine (see Kaplan, 1995), or even experiences of beauty as part of an aesthetic experience (Fancourt \& Finn, 2019; Mastandrea et al., 2019).

\section{Digital, Online Art—Would this translate to similar Wellbeing impacts? (RQ1)}

Online and digital technology might offer a natural ability to further empower the use of the arts in health and wellbeing formats. Today, art viewing, cultural engagements, and trips to art or other types of museums can take place via computers, tablets, smartphones and even virtual reality. This would certainly make it possible for many more individuals to engage art $(\mathrm{Bu}$ et al., 2021; Leng et al., 2014). Personal media, bringing artworks directly to a viewer could perhaps duplicate many of the same results, especially if beneficial effects are tied to the visual or cognitive-emotional aspects of experience. The potential for targeted, short bite-size interactions, with augmented information, or even art seen in the comfort of one's personal space without need for extra effort or distractions, perhaps could overcome some of the issues often given for why individuals may not enjoy art (Pelowski et al., 2014), allowing art to be more accessible, understandable, personal, even increasing impact beyond traditional installations or museum visits (Alelis et al., 2015). 
At the same time, a long-running argument in especially art-critical, and more recently, psychological discussions suggests that art, for its full effect, might require to be seen in person, and that digital formats or other reproductions lose necessary aspects_immediacy, ambiance, level of engagement or importance, even artwork size — of the experience (Benjamin, 1968; Berger, 2008; Pelowski, Forster, et al., 2017; Specker et al., 2021). A handful of studies have suggested that art especially in digital formats, when compared to in-person gallery viewing, may lead to lower ratings of pleasantness (Locher et al., 1999, 2001; Locher \& Dolese, 2004), interest (Locher et al., 2001; Locher \& Dolese, 2004), liking, time spent viewing (Brieber et al., 2014) or even positive emotion or arousal (Brieber et al., 2015), all of which might be important for wellbeing benefits. Beyond this, if art impacts are driven more by the tangential aspects of an in-person visit - making a special trip, being with other people; even taking a break from everyday life activity or physical exertion itself (McLean, 2011) — these might be lost or diminished in online formats, or may connect to different results, for example, with factors such as life satisfaction or loneliness. ${ }^{1}$

The ability for online engagements to lead to wellbeing-related impacts is especially in need of research. To our knowledge, only two studies have even begun to provide tentative evidence. Leng et al. (2014) compared cultural activities (cooking and craft exercises) via tablets to traditional in-person activities, finding that participants displayed similar or better levels of wellbeing in the tablet group. Tyack et al. (2017) actually examined if art interventions could be delivered through touchscreen tablets. Assessing dementia patients in their homes; wellbeing

\footnotetext{
${ }^{1}$ Interestingly, although one might surmise that a solution to loneliness requires being with other people or engaging at least in communal activity, one of the topics specifically addressed in Covid-19 times-related online art was loneliness, with several exhibitions featuring works on this theme (Jones, 2020; Knott, 2020).
} 
(happiness, wellness and interestedness, 1-100 scale) was measured before and after freely viewing a set of artworks (with the ability to select and move between styles) via a specialized app designed for the study. The results showed that after each session of around 20 minutes, wellbeing increased, although not significantly. These papers conducted their studies with specialized samples of dementia patients, and as part of therapy programmes (see also Zubala et al., 2021 for research on digital creative art therapy). Even in-person interventions have, somewhat surprisingly, not typically considered art brought into individuals' homes. However, an ongoing mental health survey conducted by University College London in the UK (Covid-19 Social Study, 2020) found, more than a fifth of 70,000 respondents engaged more with the arts during lockdown than before. Those individuals who identified as having a mental illness or disability were likely to engage more in the first 22 weeks, which was speculated to be due to the move of cultural institutions online (Bu et al., 2021), however this study did not quantify whether such use translated to actual effects.

\section{'Art' vs. 'Non-Art' Cultural Engagements? (RQ2)}

The above issues touch another aspect that we explore in this paper, regarding how engagements with art might impact individuals via online formats in comparison to other varieties of cultural engagements. On one hand, engaging with 'art' might be an ideal means to induce pleasurable and enjoyable experiences (Pelowski, Gerger, et al., 2017). Previous research has found that viewing objects that one believes to be 'art' (either via external cues or labels or derived from personal opinion) can lead to higher liking, beauty, pleasure (Leder et al., 2004; Locher et al., 2001; Pelowski, Gerger, et al., 2017), positive affective experiences (Cupchik et al., 2009; Kirk et al., 2020), and even elicit greater activation of reward-related brain areas (Kirk et al., 2009; Lacey et al., 2011), all of which may lead to greater wellbeing or mood benefits 
(Becker et al., 2019; Berridge \& Kringelbach, 2011). Having stronger aesthetic experiences, which is noted as a possible wellbeing mechanism (Fancourt \& Finn, 2019), are likely to be particularly pronounced with art.

On the other hand, wellbeing impacts can be tied to many other types of cultural engagement. For example, the in-person research referenced above is inclusive of a broad range of, often overlapping, cultural activities (see Davies et al., 2016). In just museum visits, one can find, among others, history, or science museums, which have been linked to wellbeing (Wheatley \& Bickerton, 2017, 2019). Research has found positive impacts from the simple presence of other visual materials such as photos of nature (White et al., 2010). However, most research to date has not considered the impact of art in direct comparison to other types of cultural engagement in terms of health and wellbeing, and such distinction and comparison is argued to be a target for research (Ander et al., 2013). This is especially true with the internet, where there is a wealth of activities that offer potential wellbeing benefits (e.g., Son \& Lee, 2021 for a recent study on stress and online shopping).

\section{Exploratory Factors-One Size Might Not Fit All}

Finally, in addition to our main research questions, several other dependent and independent factors might be important in assessing the impact of online art. First, outcomes likely depend on differing aspects of the individual's experience. Research suggests that individuals' reactions to art, and likely all forms of culture, are quite subjective (Pelowski, Gerger, et al., 2017). Most basically, regardless of what kind of cultural engagement, this involves aspects such as whether one would like to visit again, finds beautiful or meaningful what they are engaging with. 
Similarly, research has shown that — at the emotional and cognitive level—individuals respond differently to the same artwork. For example, the extent to which an engagement with art is more or less intellectually stimulating, educational, novel, harmonious, or boring, among other felt emotions (Pelowski, Markey, et al., 2017 for review), might also differentiate impacts to wellbeing.

Further, there may be specific relationships between the different aspects of wellbeing outcomes (i.e. mood, anxiety, loneliness), which also help to define the mechanisms underlying interventions. For example, past research has suggested that some aspects such as mood or stateanxiety may be particularly variable to immediate fluctuations in the environment, like an art intervention (e.g., Fredrickson \& Branigan, 2005). These factors may then subsequently contribute to changes in more complex cognitive states such as loneliness and evaluative aspects of subjective wellbeing or life satisfaction and call for consideration.

This study also raises basic questions about dose and duration. For example, how much time and how much art is enough to see a positive impact? - might effects be found with even one brief interaction with one work of art?

\section{Method}

\section{Participants}

To test the above questions, the study included a final sample of 84 participants (65 women, 17 men, 1 other, 1 unknown; $M_{\text {age }}=34.89, S D=14.47$, range $=21$ to 74 ). Participants were further divided between two conditions of cultural engagement, with 40 individuals (31 women, $M_{\text {age }}=35.40, S D=14.44$, range $=22$ to 74 ) exposed to an 'art' condition (see below) and 44 (34 women, $M_{\text {age }}=34.43, S D=14.64$, range $=21$ to 72 ) exposed to a 'non-art' condition. Participants were recruited as a convenience sample through social media and word-of-mouth 
and consisted of nationals from Europe (43\%), the Americas (48\%), Asia (8\%), and Africa (1\%). They received no compensation for participation. They engaged in the study between April 17 to June 6, 2020 during the first wave of the Covid-19 pandemic when most nations had instituted various degrees of lockdown.

The final sample was derived from an initial sample of 143, with participants removed due to exclusion criteria (see Results). The study followed the ethical standards of the Declaration of Helsinki and the University of Vienna ethics board.

\section{Stimuli}

The online cultural engagement conditions consisted of two interactive exhibitions, broadly argued to be generally thought of as 'art' and a 'non-art,' counterbalanced between subjects. ${ }^{2}$ See Figure 1 for stimuli examples and Supplementary Materials for an access link and all accompanying texts. Both sets of stimuli were selected from Google Arts and Culture, a free online repository of virtual art galleries, interactive experiences, and educational materials on a range of topics covering culture, history, and art (About Google Cultural Institute, 2020), and consisted of a single visual image which could be appreciated by itself, as well as zooming in on various details accessed by scrolling with the mouse. Though the order of details was predetermined, the participants had the autonomy to scroll up and down as they liked. Each detail was accompanied by related text (e.g., “...This is one of 18 canvases of this view in differing light conditions..."). Thus, in both cases they were aimed at duplicating what might be

\footnotetext{
${ }^{2}$ Note, we are not making any ontological claim regarding the definition of art, however we selected our stimuli so the painting was an example of objects highly popular and well-known to the general public as fine art, whereas the non-art condition had no clear connection to art and was focused on a different aspect of culture, namely the cultural/history of food.
} 
an engagement with a single painting or exhibit, where one might move forward or backwards and read accompanying labels or materials.

For the 'art' condition (hereafter, 'Water-lilies condition'), we used an exhibition of Monet's The Water-Lily Pond; An in-painting tour from the National Gallery, London (Google Arts and Culture, 2020). This displays the single painting (The Water-Lily Pond, 1899) of a bridge over a water-lily-festooned pond in Giverny, and was chosen because landscape and waterscape artworks have been suggested to be especially useful to reduce stress and anxiety among both patient and non-patient populations (Ulrich \& Gilpin, 2003). The artist, Claude Monet, an Impressionist painter, also himself proposed that the rooms in which his water-lily paintings hang are asylums of peaceful meditation (White, 1978). The accompanying text in each frame focused on painting features (colour, brushstrokes) or contextual information about the artist and painting.

The 'non-art' stimulus (hereafter, 'Bento condition') was A Bitesize History of Japanese Food; Explore a mouthwatering box of Japan's iconic cuisine (Google Arts and Culture, 2020). This explored a diagram in the shape of a bento box, containing photos and facts introducing the viewer to the history and traditions of Japanese food, and included images of food and foodrelated activities, such as harvesting or drinking. The text covered aspects of Japanese culture, including origins of specific dishes, agricultural traditions, and food preparation.

$<$ Figure $1>$

\section{Procedure}

The study employed a mixed Two (Time: pre vs. post, within-participant) x Two (Online Cultural Engagement: art vs. non-art, between-participant) design. Participants were supplied with a survey link via Qualtrics, which led them to a short description of the study. The 
description noted that the study was designed to test participants before and after they freely explored an online exhibit, and the types of experiences that could be engendered. After agreeing to participate, participants were first asked to provide demographic information, and then completed a pre-viewing baseline measure of our dependent variables. Upon beginning this, participants were also already assigned, unbeknownst to them, to one of the two types of online engagements, with the general description being the same for both the Water-lilies and Bento conditions. In both cases, we refrained from using the term 'art' but rather referred to an 'online experience' or 'exhibit' in all instructions. Participants were explicitly instructed not to enter any other parts of the website or other webpages and to return to the post survey once finished. They were then presented with a hyperlink button which, upon clicking, would open either the art or non-art Google Arts and Culture exhibition in a new window, where they were able to spend as much time as they liked viewing and interacting with the stimuli.

\section{Pre-viewing Survey - Demographics and Personality, and Covid Related Conditions}

In the pre-study survey, participants provided demographic descriptions (age, gender, nationality, location of current residence) as well as the status of their lives during the present lockdown, including lockdown severity (ranging from 'I am staying only in my personal residence' to 'I am not in lockdown at all', see Supplementary Materials Table S1), duration, and number of other people living in the participant's residence. The Ten Item Personality Inventory (TIPI) was used to assess personality traits (Gosling et al., 2003). Art expertise was assessed through four items derived from the Vienna Art Interest and Art Knowledge Questions (Specker et al., 2020) on art education or training, art interest, and art related behaviour. We also asked if participants had ever visited any online art presentation previously in their lives. 


\section{Pre-/Post-viewing Survey-Wellbeing, Anxiety, Mood, Loneliness}

To measure the impact of visiting the online material, we assessed six dependent variables (hereafter 'Wellbeing DVs'). These were presented in both the pre- and post-viewing survey to assess change after the brief online engagement; (1) The De Jong Gierveld 6-Item Loneliness Scale (De Jong Gierveld \& Van Tilburg, 2010); (2) State-Trait Anxiety Inventory (Marteau \& Bekker, 1992); (3) The Satisfaction with Life scale (Diener et al., 1985); (4) The Subjective wellbeing scale (Tinkler \& Hicks, 2011); (5-6) Mood was assessed by two questions asking participants to rate their overall positive and negative mood. Note, all Likert-type scalebased questions here and in the post-study were adapted to seven points to aid consistency of answering.

\section{Online Experience Evaluations}

In the post-viewing questionnaire, participants also appraised the stimuli via four questions on a 7-point scale ( 1 = 'not at all,' 7 = 'very much'), indicating how beautiful, meaningful, and good the stimuli were, and how much they would like to visit the website again. Participants were also asked if they felt they had seen 'art' during the experience or not (binary forced-choice: 'I saw art'/ 'I saw something else').

As an exploratory means of collecting more information on the nature of the experience, participants were presented with a list of 55 cognitive-emotional terms (e.g., "serenity", "bored", “angry", and "harmonious," etc.), and asked to express how much they felt each of them while they were viewing the stimulus $(1=$ 'not at all,' $7=$ 'very much'). See Supplementary Materials Table S2 for full list. Previously, variations of this list have been used to further examine types of art experiences (Pelowski, 2015; Pelowski et al., 2018, 2019). 


\section{Results}

Participants were included in the final sample if they had completed more than $65 \%$ of the total survey, allowing at least one pairwise comparison, and if they had spent at least 10 seconds viewing the online materials. Forty participants were excluded due to insufficient survey completion; 19 were excluded due to overly short viewing times.

Supplementary Materials Table S1 provides the demographic and art interest descriptive statistics across the final sample of participants divided between conditions, as well as betweenconditions statistical comparisons. A comparison of individual's average art interest, typical art engagement, and art training found no significant differences between groups (Table S1). In addition, there were no significant differences in days spent in lockdown $(M=50.6)$, age, or personality.

Here, and throughout the results section, we employed a mix of parametric and equivalent non-parametric analyses on a case-by-case basis, depending on violation of assumptions. These are indicated where applicable.

\section{Descriptive Results of Online Engagements-Viewing Time, Appraisal, Experience}

Mean results of reported appraisal and cognitive-emotional terms are shown in Figure 2 (see also Supplementary Materials Table S2 for descriptive statistics). Comparison between conditions for whether individuals believed they had seen a work of 'art' versus 'something else'

revealed, as expected, a significant difference $\left(\chi^{2}(1,84)=5.25, p=.02\right)$, with $92 \%$ of individuals in the Water-lilies condition agreeing they saw art, compared to $72 \%$ in the Bento condition (this, a still rather high result, is also considered further below).

Participants in both conditions, on average, evaluated their online engagements positively, with Wilcoxon rank-sum tests conducted for each variable independently revealing 
comparatively higher beautifulness and goodness appraisals in the Water-lilies condition $(Z=$ 4.18, $p=<.001$, and $Z=-4.56, p=<.001$ ), whereas meaningful and desire to visit again did not show differences $(Z=-1.81, p=.279$ and $Z=-2.06, p=.157$, respectively; $p$ values adjusted with a Bonferroni correction for multiple comparisons; Non-parametric tests employed due to violation of normality of appraisals' distributions, see Figure S2 of Supplementary Materials). The four appraisals themselves also showed positive intercorrelation $\left(\mathrm{r}_{\mathrm{s}}=.55\right.$ to .71$)$ across conditions.

$<$ Figure $2>$

On average, participants engaged with the online content for around one-and-a-half to two minutes (Water-lilies: $M=107 \mathrm{~s}, S D=69 \mathrm{~s}$, range $=11$ to $274 \mathrm{~s}$; Bento: $M=209 \mathrm{~s}, S D=$ $249 \mathrm{~s}$, range $=10$ to $1401 \mathrm{~s})$. Notably, as can be seen in the ranges, even the longest times spent viewing only approached about 4.5 minutes. A Wilcoxon rank-sum test comparing conditions was not significant $(Z=-1.76, p=.08)$. Across conditions, viewing time was also not correlated with the four appraisals of the material themselves (all $r \mathrm{~s}=.04$ to .1 , see Figure 4 ).

When asked how they felt while engaging the online content, participants indicated quite similar positive-valence emotions and cognitive states (e.g., serenity, happy, stimulated, insight), with low levels of negative emotions (fear, embarrassed, anger, etc.), in both conditions (see Figure 2 for comparisons at the individual item level).

\section{Did Art/Non-Art Online Engagement Impact Wellbeing?}

Descriptive statistics for the Wellbeing DVs, pre- and post-viewing, are provided in Table 1 and visualized as violin plots with individual slopes for each participant, in Figure 3. Effect sizes for each condition and the total sample are shown in Figure 3. Correlations between 
Wellbeing DVs (pre-, post-, and post-pre changes) are shown in Figure S1 of Supplementary Materials.

As can be seen, in general, participants showed mean baseline scores (before viewing) at just below the midpoints for most DV scales, albeit with a rather large spread across participants. We also found a general moderate correlation between most baseline Wellbeing DVs (Supplementary Materials Figure S1). No differences in baseline scores were found, for all Wellbeing DVs, when comparing between condition groups (Table S1).

In general (Figure 3) the Wellbeing DVs, when considered after the online engagements, tended to show mean changes in the expected directions (i.e., decreased state-anxiety, loneliness, negative mood; increased positive mood and wellbeing), with the Water-lilies conditions tending to have generally larger effect sizes.

$<$ Figure $3><$ Table $1>$

\section{General Impact Across Wellbeing DVs and Between Conditions}

To statistically test the results, we first conducted a two-way mixed repeated measures multivariate analysis of variance (MANOVA) on the six Wellbeing DVs, with Time (pre, post; within-subjects) and engagement Condition (Water-lilies, Bento; between-subjects) as independent variables (conducted in R studio, R version 3.6.2; RStudio Team, 2019). In MANOVA, group differences are tested by creating a linear combination of the Wellbeing DVs that maximize group differences, and is considered to be optimal when Wellbeing DVs show correlation (Tabachnick \& Fidell, 2013). This was conducted on a reduced sample of 78 participants (36 Water-lilies, 42 Bento) due to three participants having missing data and the detection of three multivariate outliers (2 Bento, 1 Water-lilies) with Mahalanobis distance exceeding the critical value $(22.46 ; \mathrm{df}=6, \mathrm{MD} 1=23.35, \mathrm{MD} 2=24.52 .896, \mathrm{MD} 3=24.81)$. 
Analysis including the outliers however did not substantially differ, however, it impacted the marginal significance of a covariate in one of the post-hoc analysis (see Table 2). The Wellbeing DVs were not multivariate normally distributed, however MANOVA has been shown to be robust to violations of the assumption of multivariate normality when the sample is, as in our case, $>30$ per condition (Tabachnick \& Fidell, 2013). The data met assumptions for homogeneity of variance (all Levene's tests $p>.05$ ) and multicollinearity for Wellbeing DVs (correlations between Wellbeing DVs were <.8).

The analysis showed a main effect of $\operatorname{Time}\left(F(6,71)=3.65, p=.003 ; \eta_{p}^{2}=0.24,90 \% \mathrm{CI}\right.$ $=[0.05 ; 0.31])$ across both conditions. However, neither the main effect of Condition $(F(6,71)=$ $0.56, p=.763)$ nor the Condition $x$ Time interaction $(F(6,71)=0.64, p=.700)$ significantly affected the linear combination of Wellbeing DVs. These results suggested that both types of online cultural engagement significantly impacted the combined Wellbeing DVs, however there was not a significant difference between the impact of the Water-lilies and Bento conditions $\left(\eta_{p}^{2}\right.$ $=0.05,90 \% \mathrm{CI}=[0 ; 0.07])$. An additional series of repeated measures ANOVAs, run independently for each of the Wellbeing DVs and looking at potential differences between Condition, showed all interaction with Time not to be significant, with $p$-values ranging from $p$ $=.171$ to $p=.971$ (all $p$-values uncorrected, see Supplementary Materials Table S3).

\section{Specific and Shared Impact on Individual Wellbeing DVS}

To further consider the impact of the online interventions on the specific Wellbeing DVs which were assessed above, as well as their potential interrelation, we carried out a RoyBargman stepdown analysis. This approach is suggested as a best-practice follow up for MANOVA (Tabachnick \& Fidell, 2013), especially in cases of correlation between Wellbeing 
DVs as noted above. This procedure consisted of, first, univariate post-hoc ANOVAs on each individual DV, in order to examine main effects of time at the individual DV level.

This was followed by a set of ANCOVAs, which tested for the effects of Time on each of the Wellbeing DVs, taking into account other Wellbeing DVs as covariates in a prioritized, step-down order. This second step allowed us to control for effects that are shared between the Wellbeing DVs, a phenomenon found in past studies looking at factors of wellbeing (i.e. Cohen et al., 2017; Dua, 1993). As a consequence of the above findings of a significant impact of the online engagements across both conditions with a non-significant difference between them, the samples were combined for these two subsequent analyses. All independent $p$-values for each of the main effects in the following tests were corrected for the number of comparisons $(\mathrm{N}=6, p$ values for covariates were not corrected).

At the univariate level, the results, reported in Table 2 (see also Figure 3 for effect sizes), indicated that the online cultural engagements had a significant impact on four of the Wellbeing DVs - negative mood, state-anxiety, subjective wellbeing, and loneliness - but not on positive mood or satisfaction with life.

For the stepdown analysis, the effects of the online engagement on the Wellbeing DVs were prioritized as follows: (1) effect on mood (negative and positive); (2) effect on negative variables (state-anxiety and loneliness), after having controlled for the effect of mood; and (3) the effect on positive wellbeing variables (satisfaction with life and subjective wellbeing) after accounting for changes in mood and negative variables. This order was selected because, as noted in the introduction, changes in mood and state-anxiety are argued to be potentially more variable as a result of changes in one's immediate environment, which can possible contribute to 
changes in more complex cognitive states (Fredrickson \& Branigan, 2005). Homogeneities of the regression slopes were obtained for all of the subsequent univariate analyses.

The results suggested that changes observed in state-anxiety and wellbeing were not uniquely impacted by the online cultural engagements beyond the shared effect on mood. For loneliness, although significantly impacted by the online engagements, the effect was accounted for by changes in state-anxiety, after controlling for the effect of changes in mood.

$<$ Table 2>

\section{Exploratory Analyses}

We then turned to a post hoc exploratory analysis, considering some of the other research questions noted in this study, as well as some of the above-reported findings.

\section{Viewing time and Appraisal of Experience}

First, as a general consideration of the relation between the nature of experience and its impact, we considered the correlation between appraisals of the online material (meaning, beauty, goodness, desire to visit again) and the change scores (post-pre) for the individual Wellbeing DVs. We similarly considered viewing time. Due to the violations of normality, we used Spearman's Rank correlations. Results for combined online conditions are shown in Figure 4. Results split between conditions can be found in Supplementary Materials Table S4.

$<$ Figure $4>$

Decreases in especially negative mood, as well as state-anxiety and positive mood, were significantly related to relatively higher appraisals. Changes in negative mood were related to reported desire to visit the experience again, meaningfulness, beauty, and marginally related to goodness. Similarly, changes in state-anxiety and positive mood were significantly related to desire to visit again, goodness, with state-anxiety also marginally related to beauty. Goodness 
alone also was correlated with lower loneliness. Note however that, only the relationships between meaningfulness and changes in negative mood and goodness and changes in loneliness remained significant or marginally significant (adjusted $p s=.049$ and .067 , respectively; Bonferroni correction for 24 tests).

Interestingly, while both conditions showed the same general directionality of effects, the effect sizes of the relationships were much larger in the Water-lilies condition, while assessment of the Bento condition group did not have most results reach significance.

Also interesting, amount of time spent by individuals was not significantly related with any DV change $\left(r_{s}=-.21\right.$ to .19$)$.

\section{Seeing 'Art'}

We then also followed up on participants' classification of the stimuli as 'art,' especially considering the large number of individuals that considered even the Bento condition to be an artwork. A breakdown between participants who said they saw either 'art' or 'something else,' regardless of their actual Water-lilies/Bento assignment, comparing appraisals of beauty, goodness, meaningfulness, and desire to visit again are shown in Table S5. A breakdown of DV change scores for 'I saw art' and 'something else' is shown in Table S6. Difference in appraisals and Wellbeing DVs are shown in Figure 4. To consider these distinctions, we used multivariate analysis with groups, 'I saw art' group $(n=63)$ or 'I did not see art' $(n=15)$. Wilcoxon rank sum test suggested that participants who indicated that they saw 'art,' regardless of assigned condition, reported significantly higher meaningfulness $(Z=-2.29, p=.022)$, beauty $(Z=-3.72$, $p<.001)$, and goodness $(Z=-3.99, p=<.001$, see also Table 3$)$, and marginally significant higher desire to visit again $(Z=-1.91, p=.055)$. 
However, at the same time, a series of two-sample nonparametric Welch's t tests, comparing change scores between conditions, did not reveal significant differences between those who reported seeing 'art' or 'something else' for any DV (all p-values >. 26).

$<$ Table 3>

\section{Cognitive-Emotional Factors in Experience}

Finally, we returned to the list of the cognitive-emotional items that participants reported feeling during their experiences. To reduce the data and to identify potential patterns, we conducted a Principal Component Analysis (PCA) on all 55 items. A parallel analysis using Monte Carlo simulation with permutations (5000) of the raw data set, conducted in SPSS with the rawpar.sps script (O’Connor, 2000), suggested four significant components. These were then assessed using a Direct Oblimen rotation, which allows for an orthogonal or oblique solution, expected to provide a more natural fit for the data. The Kaiser-Meyer-Olkin measure verified sampling adequacy $\left(\mathrm{KMO}=0.65 ;\right.$ Field, 2009). Bartlett's test of sphericity $X^{2}=3359.9, p<$ 0.001, also indicated sufficient correlations between items to support the PCA analysis.

The resulting rotated components solution (Supplementary Materials Table S7) accounted for $48.48 \%$ of the variance, with a breakdown of items more or less in line with past findings (Pelowski, 2015; Pelowski et al., 2019). The first component (26.20\% of variance) largely consisting of items related to positive or socially-focused terms (highest loadings: confident, changed self-image, compassion, gratitude, free, sublime, etc.). The second component (11.11\%) described largely negative and again socially-focused terms (fear, like crying, sad, powerless, stress, shame, guilt, vulnerable). The third component $(5.91 \%)$ included items previously suggested to indicate largely "facile" (Pelowski, 2015) or unrewarding experience (disappointed, bored, need to leave, embarrassed, confused). The fourth component 
$(5.30 \%)$ described more insightful or transformative aspects (absorbed, thrilled, insight, change mind, epiphany, etc.).

Component scores were then calculated per component for each participant (via regression method). These scores were then used in a series of multiple regressions to predict change in the Wellbeing DVs. See Supplementary Materials Tables S8-S10 for all results both considering combined or split between conditions. Once again, changes in especially negative mood emerged as being significantly predicted by the combined model $(F(4,69)=6.29, p$ $\left.<.001, R^{2}=.27\right)$. Further analysis revealed that such changes were significantly driven by Component 1 ('positive/social affect'; $t=-2.15, p=.035$ ), with higher scores relating to a decrease in negative mood, and to lower scores of Component 3 ('facile'; $t=3.75, p>.001$ ).

Decreasing state-anxiety was also significantly predicted, again, by component one $(t=$ $2.39, p=.020$; main model $\left(F(4,69)=2.285, p=.069, R^{2}=.12\right)$. No other Wellbeing DVs were significantly predicted by any of the components ( $p$ values are uncorrected). Interestingly, as with the appraisals above, the relationships between the cognitive-emotional components and Wellbeing DVs also tended to emerge more saliently in the Water-lilies condition, whereas with the Bento condition no significant state-anxiety or mood-related effects were found.

\section{Discussion and Conclusion}

We found a significant impact on several wellbeing variables in a pre-/post-paradigm in which individuals were asked to briefly visit an online art exhibition (one Monet painting) or another similarly-formatted, 'non-art' cultural engagement (a display of Japanese culinary tradition, aligning more to what might be encountered in a history museum offline). This included lowered state-anxiety, negative mood, loneliness, and increased subjective wellbeing. This occurred, in the art condition, with an interactive display of one painting, and in the other 
display of cultural material, and in both cases, over durations averaging one-and-a-half to two minutes, but ranging from 10 s to 4.5 minutes, notably with no significant relationship between positive impact and actual time spent engaging.

A stepdown analysis found that the changes in wellbeing dependent variables (wellbeing DVs) tended to be explained by changes in negative mood (in the case of positive mood, stateanxiety and wellbeing), while improved loneliness was explained by improvements in stateanxiety. Finally, the 'art' condition had generally larger effect sizes, but was not significantly 'better' than 'non-art' at improving any wellbeing DV. When considering the nature and appraisals of the experience, a significant relation was found between decreases in negative mood, and to a lesser extent state-anxiety and positive mood, and positive appraisals of the experience (desire to visit again, beauty, and, most pronounced, meaningfulness). Similarly, changes in negative mood and state-anxiety related to higher reported positive or sociallyfocused cognitive-emotional states (Component 1).

\section{RQ1—general efficacy for online art and cultural interventions}

These results raise several implications for both pragmatic and theoretical application of the arts for wellbeing. First, this provides initial evidence that such wellbeing effects can be found via brief interactions with art and similar cultural materials online, impacting many of the same variables as considered in in-person studies. This finding, detected with an adult sample of convenience, during a unique Covid-19-induced lockdown period, might be viewed as proof of concept that technology, via the internet, can deliver targeted doses of art and culture into the everyday lives of individuals. The findings also add evidence to the potential of digital reproductions to produce similar effects as that found with real artworks installed in common spaces or seen in-museum visits. 
The reduction of reported loneliness also merits specific mention. While probably only tangentially connected to the art experience — via the changes in anxiety - a claim could be made that the loneliness-reducing aspects of cultural engagements, routinely found in-person (Todd et al., 2017; Tymoszuk et al., 2019), might come about from the social connection or collaborative nature of visiting a museum or sharing art experiences. Here, however, we found that even visiting an exhibit, likely by oneself via the internet, led to significant effects. Possibly due to the communal nature of visiting a public website and seeing another person's art or design. In support of such a communal claim, loneliness was one of the only variables that showed slightly larger effects in the Bento condition, which focused on human activities in a more explicit way. This adds credence to recent initiatives, especially arising during Covid-19, for online arts and culture exhibitions to target loneliness during isolation from others - another compelling target for future research.

When comparing the effect of online art interventions to past literature, our findings are similar. Looking to the two most salient effects found in this paper-improvement in mood and state-anxiety — we found moderate to large effects in the art condition (negative $\operatorname{mood} \eta_{p}^{2}=.12$, positive $\operatorname{mood} \eta_{p}^{2}=.17$, and state-anxiety $\left.\eta_{p}^{2}=.19\right)$. These are in line, for example with Ho et al. (2015) who reported a pre/post change (assessed via Brief Mood Introspection Scale) following an art exhibition visit in a hospital. As well as Paddon et al. (2014), who reported medium effect sizes in negative mood (Cohen's $d=0.38)$ and positive mood $(d=0.69)$, assessed via Positive and Negative Affect Schedule (PANAS), following a cultural museum object-handling session in a hospital (also see Clow and Fredhoi’s (2006) report of reduction in self-reported stress after individuals spent a lunch break in-person in a museum of art). 
Even comparing to other domains, our results remain roughly equivalent. For example, Cracknell et al. (2016) reported a change in affective valence (assessed via 11-point bipolar Feeling Scale), following a roughly five-minute intervention in which participants observed an aquarium fish tank, $\eta_{p}^{2}=.470$ (negative-to-positive). Comparing across interactions with nature, which have been routinely shown to provide impacts on mood, a meta-analysis (McMahan \& Estes, 2015) of both lab or in-person interventions reported a standardized reduction in negative affect across studies of $r=.12$ (increase in positive affect $r=.31$ ). In emerging results of other online interventions, Howells et al. (2015) report a smartphone-based mindfulness intervention impact on affect (measured via PANAS after 10 days of use) of medium size effect $(\eta 2=.071)$ for positive affect and small effect $(\eta 2=.010)$ for negative mood. Keeping in mind the selfreport method of the present study, differences in assessment scales, and leaving open the question of the duration of impact or whether self-report might relate to physical or behavioural differences, our results provide compelling evidence for art and culture in online spaces.

\section{RQ2-Is there anything different about engaging art versus other cultural content?}

In regards to our second research question, we found mixed, albeit still compelling suggestions about the potential difference that 'art' might play in improving wellbeing. We did not find a significant difference between conditions. However, we did find larger effects in the case of art. One probable explanation for the lack of difference is that both conditions, which were selected to be formally similar, evoked similar responses_-for example, a generally positive interaction with visually pleasing material. Note, while the artwork was rated as more beautiful and good, both conditions were equally meaningful and evoked a desire to experience them again. 
One telling result in line with the above argument was that, despite explicitly not labelling it as such on our part, we found almost $75 \%$ of those in the Bento condition actually believed that they had engaged with 'art'. This finding that individuals would label somethingeven if not intended to be seen as art in a classic sense - as an 'art' example, is in-line with past research. For example, Pelowski, Greger, et al. (2017) reported this with photographs of a number of random every-day objects, suggesting that individuals may use 'art' as a general label of particularly appreciated visual stimuli. Thus, it is reasonable to assume that we were not in fact comparing apples to oranges as we had designed, but more likely that we were comparing apples to a different variety of apples.

The fact that participants reported higher appraisals in the case of the artworks suggests that this design indeed did elicit slightly more positive responses. On the one hand, it is important to note that, the subjective 'art'/'not-art' label provided by the participants for their interventions (regardless of the actual assigned condition), did not result in significant differences between the impact of the cultural engagement at the level of our Wellbeing DVs, regardless of the higher appraisals. Thus, the label of art alone does not appear to be the a necessarily important factor of cultural interventions but rather the subjective experience of the content engaged with is a topic for future research.

\section{Duration and Dosage}

Another interesting finding of the study involves the required time and amount of art. Unlike previous studies that have typically either installed some works of art and made follow up assessments over an extended duration or asked individuals to have a complete museum experience, in the present study we found meaningful effects even with an interactive exhibition of one painting, and from a few seconds to just under five minutes. This viewing duration, aligns 
with typical ranges of individual artwork engagement in museums (Pelowski, Forster, et al., 2017). The results are also in line with other wellbeing findings. For example, the Cracknell (2016) study of aquarium-viewing found that peak impacts on mood tended to be delivered within the first five minutes. Our results provide a suggestion for the possibility of 'microdosing' art and culture that might fit into everyday routines, delivered online.

\section{Potential Mechanisms for the modulation of Mood, Anxiety, and Wellbeing}

Our study also sheds light on potential underlying mechanisms for how art can lead to wellbeing impacts. Our stepdown analysis highlighted that online cultural engagement might especially be an avenue to regulating mood, which possibly drives other positive impacts (stateanxiety, subjective wellbeing). This highlight of mood regulation is supported by past research, which has found emotion and mood regulation as a common beneficial outcome of a variety of types of arts and cultural engagement (Fancourt et al., 2019, 2021; Ivcevic \& Brackett, 2015).

The significant relationships between the impacts on mood and individuals' appraisals and cognitive-emotional experience adds new data to our understanding of how art interventions might work. The finding that higher positive cognitive-emotional states (e.g., joy, and social terms such as confident, compassion, gratitude) and more profound states such as free, moved, awe, sublime, raise interesting implications for past arguments that contributors to wellbeing impacts beyond mere pleasure. For example, many of these terms could be argued to overlap with Kaplan's (1995) discussion of "fascination" as a pillar of restorative effects from viewing nature. Empirical evidence for such nuanced aspects in art engagement is only recently emerging (Pelowski, Markey, et al., 2017), however it seems plausible that encouraging specific types of cognitive-emotional states, perhaps through choice of content, is an important consideration when designing interventions and is another target for further research. 
Several of these states (awe, moved) are also suggested to be prototypical emotions in aesthetic experience (Fingerhut \& Prinz, 2020). Along with our findings of the relationship of beauty, meaningfulness, and other positive appraisals to individual's change in mood, one could make a claim that the present results support arguments highlighting aesthetic experiences as an important factor in art interventions (Fancourt \& Finn, 2019; Mastandrea et al., 2019; Sachs et al., 2015). Fancourt and Finn (2019), include aesthetic experience in their summary of the mechanisms underlying the impact of art engagement on health and wellbeing while Mastandrea et al. (2019) further note, that aesthetic experiences from visual art can induce highly pleasurable states and these states can impact affect and aid mood regulation. In future research, these features of art and cultural interventions need to be further teased apart so that we may understand what types of experiences, and which aspects of those experiences can impact which types of individuals.

\section{Caveats and other Targets for Future Research}

This study is of course not without limitations. It should be treated as a first exploratory step, as we had a sample of convenience and little control over what type of device (computer or phone) was used, in which setting (home, office, while commuting, in hospital), and how participants engaged with the chosen stimuli (alone or with their companions). Due to the study design, we are unable to determine the causality of the relationship between appraisals, cognitive-emotional experience, and impacts on mood and anxiety. Participants first viewed the cultural content, then indicated which cognitive-emotional states they experienced while viewing, and subsequently provided their appraisals of the exhibition, followed by their actual mental wellbeing state. We would encourage further research to examine if subjective experiences such as beauty and meaningfulness, mediate the impact of said intervention on mood 
and anxiety. Or, alternately, if an improved cognitive, emotional, or physiological state influence reported appraisals. It would be interesting to compare these effects to other-perhaps less visually appealing or more information-focused — conditions, or stimuli that might not be spontaneously labelled as art but rather lacking any trace of intentionally of human connection.

That said, these results suggest that online cultural engagement, including but not limited to fine art, does seem to be a viable tool to support individuals' mood, anxiety, loneliness and wellbeing especially when such content is beautiful, meaningful, and inspires positive cognitiveemotional states in the viewer.

\section{Acknowledgements:}

We would like to thank Corinna Kühnapfel, Joerg Fingerhut, and Sandra Trupp for feedback on this manuscript and the members of the Empirical Visual Aesthetics Lab for feedback on this project. The data collection of this project was supported by the University of Vienna. Writing was funded by a grant to MP by the EU Horizon 2020 Program (No 870827). GB was supported by the BMBF and the Max Planck Society. 


\section{References}

About Google Cultural Institute. (2020). https://about.artsandculture.google.com/

Alelis, G., Bobrowicz, A., \& Ang, C. S. (2015). Comparison of engagement and emotional responses of older and younger adults interacting with 3D cultural heritage artefacts on personal devices. Behav. Inf. Technol. https://doi.org/10.1080/0144929X.2015.1056548

Allen, M., Poggiali, D., Whitaker, K., Marshall, T. R., van Langen, J., \& Kievit, R. A. (2021). Raincloud plots: A multi-platform tool for robust data visualization. Wellcome Open Research, 4, 63. https://doi.org/10.12688/wellcomeopenres.15191.2

Ander, E., Thomson, L., Blair, K., Noble, G., Menon, U., Lanceley, A., \& HJ, C. (2013). Using museum objects to improve wellbeing in mental health service users and neurological rehabilitation clients. British Journal of Occupational Therapy, 76, 208-216. https://doi.org/10.4276/030802213X13679275042645

Barbican Center. (2020, May 19). Barbican announces new content to coincide with Mental Health Awareness Week and Human Rights Watch Film Festival [Press Release]. https://www.barbican.org.uk/our-story/press-room/barbican-announces-new-content-tocoincide-with-mental-health-awareness-week

Becker, S., Bräscher, A.-K., Bannister, S., Bensafi, M., Calma-Birling, D., Chan, R. C. K., Eerola, T., Ellingsen, D.-M., Ferdenzi, C., Hanson, J. L., Joffily, M., Lidhar, N. K., Lowe, L. J., Martin, L. J., Musser, E. D., Noll-Hussong, M., Olino, T. M., Pintos Lobo, R., \& Wang, Y. (2019). The role of hedonics in the human affectome. Neuroscience \& Biobehavioral Reviews, 102, 221-241. https://doi.org/10.1016/j.neubiorev.2019.05.003 Belvedere Museum. (2020). Isolation - Sammlungsbereiche - Sammlung Online [Online Collection]. 
https://sammlung.belvedere.at/collections/219483/sitzen;jsessionid=F5863ED64B945012 8617C950900477E9

Benjamin, W. (1968). The Work of Art in the Age of Mechanical Reproduction. Illuminatioins. Trans. By Harry Zohn. Shocken Books.

Bennington, R., Backos, A., Harrison, J., Etherington Reader, A., \& Carolan, R. (2016). Art therapy in art museums: Promoting social connectedness and psychological well-being of older adults. The Arts in Psychotherapy, 49, 34-43.

https://doi.org/10.1016/j.aip.2016.05.013

Berger, J. (2008). Ways of Seeing. Penguin Classics.

Berridge, K., \& Kringelbach, M. (2011). Building a neuroscience of pleasure and well-being. Psychology of Well-Being, 1, 1-3. https://doi.org/10.1186/2211-1522-1-3

Binnie, J. (2010). Does viewing art in the museum reduce anxiety and improve wellbeing?. Museums \& Social Issues, Vol 5, No 2. https://www.tandfonline.com/doi/abs/10.1179/msi.2010.5.2.191

Brieber, D., Nadal, M., \& Leder, H. (2015). In the white cube: Museum context enhances the valuation and memory of art. Acta Psychologica, 154, 36-42. https://doi.org/10.1016/j.actpsy.2014.11.004

Brieber, D., Nadal, M., Leder, H., \& Rosenberg, R. (2014). Art in time and space: Context modulates the relation between art experience and viewing time. PloS One, 9, e99019. https://doi.org/10.1371/journal.pone.0099019

Bu, F., Mak, H. W., Bone, J. K., \& Fancourt, D. (2021). Longitudinal changes in home-based arts engagement during and following the first national lockdown due to the COVID-19 
pandemic in the United Kingdom. MedRxiv, 2021.05.14.21257233.

https://doi.org/10.1101/2021.05.14.21257233

Camic, P. M., \& Chatterjee, H. J. (2013). Museums and art galleries as partners for public health interventions. Perspectives in Public Health, 133(1), 66-71. https://doi.org/10.1177/1757913912468523

Chatterjee, H. J., \& Noble, G. (2017). Museums, Health and Well-Being (1st ed.). Routledge. https://www.routledge.com/Museums-Health-and-Well-Being/ChatterjeeNoble/p/book/9780815399537

Clayton, G., \& Potter, S. (2017). Arts on prescription: A creative and cost-effective approach to improving mental health. Arts, Health and Wellbeing: A Theoretical Inquiry for Practice. Newcastle upon Tyne: Cambridge Scholars Publishing, 160-181.

Clow, A., \& Fredhoi, C. (2006). Normalisation of salivary cortisol levels and self-report stress by a brief lunchtime visit to an art gallery by London City workers. Journal of Holistic Healthcare, 3(2), 29-32.

Cohen, J. N., Taylor Dryman, M., Morrison, A. S., Gilbert, K. E., Heimberg, R. G., \& Gruber, J. (2017). Positive and negative affect as links between social anxiety and depression. Behavior Therapy, 48(6), 820-833. https://doi.org/10.1016/j.beth.2017.07.003

Covid-19 Social Study. (2020). https://www.covidsocialstudy.org/

Cracknell, D., White, M. P., Pahl, S., Nichols, W. J., \& Depledge, M. H. (2016). Marine biota and psychological well-being: A preliminary examination of dose-response effects in an aquarium setting. Environment and Behavior, 48(10), 1242-1269.

https://doi.org/10.1177/0013916515597512 
Cupchik, G. C., Vartanian, O., Crawley, A., \& Mikulis, D. J. (2009). Viewing artworks: Contributions of cognitive control and perceptual facilitation to aesthetic experience. Brain and Cognition, 70(1), 84-91. https://doi.org/10.1016/j.bandc.2009.01.003

Cuypers, K., Krokstad, S., Holmen, T. L., Knudtsen, M. S., Bygren, L. O., \& Holmen, J. (2012). Patterns of receptive and creative cultural activities and their association with perceived health, anxiety, depression and satisfaction with life among adults: The HUNT study, Norway. J Epidemiol Community Health, 66(8), 698-703.

https://doi.org/10.1136/jech.2010.113571

Davies, C., Knuiman, M., \& Rosenberg, M. (2016). The art of being mentally healthy: A study to quantify the relationship between recreational arts engagement and mental well-being in the general population. BMC Public Health, 16(1), 15. https://doi.org/10.1186/s12889$015-2672-7$

De Jong Gierveld, J., \& Van Tilburg, T. (2010). The De Jong Gierveld short scales for emotional and social loneliness: Tested on data from 7 countries in the UN generations and gender surveys. European Journal of Ageing, 7(2), 121-130. https://doi.org/10.1007/s10433010-0144-6

Diener, E., Emmons, R. A., Larsen, R. J., \& Griffin, S. (1985). The satisfaction with life scale. Journal of Personality Assessment, 49(1), 71-75. https://doi.org/10.1207/s15327752jpa4901_13

Dua, J. K. (1993). The role of negative affect and positive affect in stress, depression, selfesteem, assertiveness, Type A behaviors, psychological health, and physical health. Genetic, Social, and General Psychology Monographs, 119(4), 515-552. 
Fancourt, D., Aughterson, H., Finn, S., Walker, E., \& Steptoe, A. (2021). How leisure activities affect health: A narrative review and multi-level theoretical framework of mechanisms of action. The Lancet Psychiatry, 8(4), 329-339. https://doi.org/10.1016/S22150366(20)30384-9

Fancourt, D., \& Finn, S. (2019). What is the evidence on the role of the arts in improving health and well-being? A scoping review (2019). Retrieved from World Health Organization website: http://www. euro. who. int ....

Fancourt, D., Garnett, C., \& Müllensiefen, D. (2020). The relationship between demographics, behavioral and experiential engagement factors, and the use of artistic creative activities to regulate emotions. Psychology of Aesthetics, Creativity, and the Arts. https://doi.org/10.1037/aca0000296

Fancourt, D., Garnett, C., Spiro, N., West, R., \& Müllensiefen, D. (2019). How do artistic creative activities regulate our emotions? Validation of the emotion regulation strategies for artistic creative activities scale (ERS-ACA). PloS One, 14(2), e0211362. https://doi.org/10.1371/journal.pone.0211362

Field, A. (2009). Discovering Statistics Using SPSS. SAGE Publications.

Fingerhut, J., \& Prinz, J. J. (2020). Aesthetic emotions reconsidered. The Monist, 103(2), 223239. https://doi.org/10.1093/monist/onz037

Fredrickson, B. L., \& Branigan, C. (2005). Positive emotions broaden the scope of attention and thought-action repertoires. Cognition and Emotion, 19(3), 313-332. https://doi.org/10.1080/02699930441000238 
Gosling, S. D., Rentfrow, P. J., \& Swann Jr., W. B. (2003). A very brief measure of the Big-Five personality domains. Journal of Research in Personality, 37(6), 504-528. https://doi.org/10.1016/S0092-6566(03)00046-1

Hansen, E., Sund, E., Knudtsen, M. S., Krokstad, S., \& Holmen, T. L. (2015). Cultural activity participation and associations with self-perceived health, life-satisfaction and mental health: The Young HUNT Study, Norway. BMC Public Health, 15(1), 544. https://doi.org/10.1186/s12889-015-1873-4

Ho, R. T. H., Potash, J. S., Fang, F., \& Rollins, J. (2015). Art Viewing Directives in Hospital Settings Effect on Mood. HERD: Health Environments Research \& Design Journal, 8(3), 30-43. https://doi.org/10.1177/1937586715575903

Howells, A., Eiroa Orosa, F. J., \& Ivtzan, I. (2015). Putting the 'app' in happiness: A randomised controlled trial of a smartphone-based mindfulness intervention to enhance wellbeing. Journal of Happiness Studies, 17. https://doi.org/10.1007/s10902-014-9589-1

International Arts and Mind Lab. (2020, August 9). A Global Call to Art: The Covid-19 Archives. https://www.artsandmindlab.org/a-global-call-to-art-archive/

Ivcevic, Z., \& Brackett, M. (2015). Predicting creativity: Interactive effects of openness to experience and emotion regulation ability. Psychology of Aesthetics, Creativity, and the Arts, 9. https://doi.org/10.1037/a0039826

Jones, J. (2020, March 27). We are all Edward Hopper paintings now': Is he the artist of the coronavirus age? The Guardian, International Edition. https://www.theguardian.com/artanddesign/2020/mar/27/we-are-all-edward-hopperpaintings-now-artist-coronavirus-age. 
Kaplan, S. (1995). The restorative benefits of nature: Toward an integrative framework. Journal of Environmental Psychology, 15(3), 169-182. https://doi.org/10.1016/02724944(95)90001-2

Karnik, M., Printz, B., \& Finkel, J. (2014). A hospital's contemporary art collection: Effects on patient mood, stress, comfort, and expectations. HERD: Health Environments Research \& Design Journal, 7(3), 60-77. https://doi.org/10.1177/193758671400700305

Kirk, U., Lilleholt, L., \& Freedberg, D. (2020). Cognitive framing modulates emotional processing through dorsolateral prefrontal cortex and ventrolateral prefrontal cortex networks: A functional magnetic resonance imaging study. Brain and Behavior, 10(9). https://doi.org/10.1002/brb3.1761

Kirk, U., Skov, M., Hulme, O., Christensen, M. S., \& Zeki, S. (2009). Modulation of aesthetic value by semantic context: An fMRI study. NeuroImage, 44(3), 1125-1132. https://doi.org/10.1016/j.neuroimage.2008.10.009

Knott, K. (2020, October 15). Online art exhibition captures pandemic scenes in Hong Kong - of loneliness, fear, but also the triumph of the human spirit. South China Morning Post. https://www.scmp.com/lifestyle/arts-culture/article/3105590/online-art-exhibitioncaptures-pandemic-scenes-hong-kong.

Kweon, B.-S., Ulrich, R. S., Walker, V. D., \& Tassinary, L. G. (2008). Anger and stress: The role of landscape posters in an office setting. Environment and Behavior, 40(3), 355-381. https://doi.org/10.1177/0013916506298797

Lacey, S., Hagtvedt, H., Patrick, V. M., Anderson, A., Stilla, R., Deshpande, G., Hu, X., Sato, J. R., Reddy, S., \& Sathian, K. (2011). Art for reward's sake: Visual art recruits the ventral striatum. NeuroImage, 55(1), 420-433. https://doi.org/10.1016/j.neuroimage.2010.11.027 
Leder, H., Belke, B., Oeberst, A., \& Augustin, D. (2004). A model of aesthetic appreciation and aesthetic judgments. British Journal of Psychology (London, England: 1953), 95(Pt 4), 489-508. https://doi.org/10.1348/0007126042369811

Leng, F. Y., Yeo, D., George, S., \& Barr, C. (2014). Comparison of iPad applications with traditional activities using person-centred care approach: Impact on well-being for persons with dementia. Dementia (London, England), 13(2), 265-273. https://doi.org/10.1177/1471301213494514

Locher, P., \& Dolese, M. (2004). A comparison of the perceived pictorial and aesthetic qualities of original paintings and their postcard images. Empirical Studies of The Arts, 22, 129142. https://doi.org/10.2190/EQTC-09LF-JRHA-XKJT

Locher, P., Smith, J. K., \& Smith, L. F. (2001). The influence of presentation format and viewer training in the visual arts on the perception of pictorial and aesthetic qualities of paintings. Perception, 30(4), 449-465. https://doi.org/10.1068/p3008

Locher, P., Smith, L., \& Smith, J. (1999). Original paintings versus slide and computer reproductions: A comparison of viewer responses. Empirical Studies of the Arts, 17, 121129.

Marteau, T. M., \& Bekker, H. (1992). The development of a six-item short-form of the state scale of the Spielberger State-Trait Anxiety Inventory (STAI). British Journal of Clinical Psychology, 31(3), 301-306. https://doi.org/10.1111/j.20448260.1992.tb00997.x

Mastandrea, S., Fagioli, S., \& Biasi, V. (2019). Art and psychological well-being: Linking the brain to the aesthetic emotion. Frontiers in Psychology, 10. https://doi.org/10.3389/fpsyg.2019.00739 
McLean, J. (2011). An evidence review of the impact of participatory arts on older people. Mental Health Foundation, Edinburgh.

McMahan, E. A., \& Estes, D. (2015). The effect of contact with natural environments on positive and negative affect: A meta-analysis. The Journal of Positive Psychology, 10(6), 507519. https://doi.org/10.1080/17439760.2014.994224

Nanda, U., Eisen, S., Zadeh, R. S., \& Owen, D. (2011). Effect of visual art on patient anxiety and agitation in a mental health facility and implications for the business case. Journal of Psychiatric and Mental Health Nursing, 18(5), 386-393. https://doi.org/10.1111/j.13652850.2010.01682.x

O’Connor, B. P. (2000). SPSS and SAS programs for determining the number of components using parallel analysis and Velicer's MAP test. Behavior Research Methods, Instruments, \& Computers, 32(3), 396-402. https://doi.org/10.3758/BF03200807

Packer, J., \& Bond, N. (2010). Museums as restorative environments. Curator: The Museum Journal, 53(4), 421-436. https://doi.org/10.1111/j.2151-6952.2010.00044.x

Paddon, H., Thomson, L., Menon, U., Lanceley, A., \& Chatterjee, H. (2014). Mixed methods evaluation of well-being benefits derived from a heritage-in-health intervention with hospital patients. Arts \& Health, 6 .

Patil, I. (2021). Visualizations with statistical details: The 'ggstatsplot' approach. Journal of Open Source Software, 6(61), 3167. https://doi.org/10.21105/joss.03167

Pelowski, M. (2015). Tears and transformation: Feeling like crying as an indicator of insightful or "aesthetic" experience with art. Frontiers in Psychology, 6. https://doi.org/10.3389/fpsyg.2015.01006 
Pelowski, M., Forster, M., Tinio, P., Scholl, M., \& Leder, H. (2017). Beyond the lab: An examination of key factors influencing interaction with 'real' and museum-based art. Psychology of Aesthetics Creativity and the Arts, 11. https://doi.org/10.1037/aca0000141

Pelowski, M., Gerger, G., Chetouani, Y., Markey, P. S., \& Leder, H. (2017). But is it really art? The classification of images as "art"/"not art" and correlation with appraisal and viewer interpersonal differences. Frontiers in Psychology, 8. https://doi.org/10.3389/fpsyg.2017.01729

Pelowski, M., Hur, Y.-J., Cotter, K., Ishizu, T., Christensen, A., Leder, H., \& Mcmanus, I. (2019). Quantifying the if, the when, and the what of the sublime: A survey and latent class analysis of incidence, emotions, and distinct varieties of personal sublime experiences. Psychology of Aesthetics Creativity and the Arts. https://doi.org/10.1037/aca0000273

Pelowski, M., Leder, H., Mitschke, V., Specker, E., Gerger, G., Tinio, P. P. L., Vaporova, E., Bieg, T., \& Husslein-Arco, A. (2018). Capturing aesthetic experiences with installation art: An empirical assessment of emotion, evaluations, and mobile eye tracking in Olafur Eliasson's “Baroque, Baroque!” Frontiers in Psychology, 9. https://doi.org/10.3389/fpsyg.2018.01255

Pelowski, M., Liu, T., Palacios, V., \& Akiba, F. (2014). When a body meets a body: An exploration of the negative impact of social interactions on museum experiences of art. International Journal of Education \& the Arts, 15(14), n14.

Pelowski, M., Markey, P. S., Forster, M., Gerger, G., \& Leder, H. (2017). Move me, astonish me... delight my eyes and brain: The Vienna Integrated Model of top-down and bottomup processes in Art Perception (VIMAP) and corresponding affective, evaluative, and 
neurophysiological correlates. Physics of Life Reviews, 21, 80-125.

https://doi.org/10.1016/j.plrev.2017.02.003

Radermecker, A.-S. V. (2020). Art and culture in the COVID-19 era: For a consumer-oriented approach. SN Business \& Economics, 1(1), 4. https://doi.org/10.1007/s43546-020-00003y

Rice, G. (2020). The Necessity of the Arts in a Time of Pandemic: How the University of Wisconsin-Madison is Responding. Division of the Arts. https://artsdivision.wisc.edu/2020/04/16/necessity-of-arts-in-pandemic/

Roberts, S., Camic, P. M., \& Springham, N. (2011). New roles for art galleries: Art-viewing as a community intervention for family carers of people with mental health problems. Arts \& Health, 3(2), 146-159. https://doi.org/10.1080/17533015.2011.561360

Romano, A. (2020, March 12). 12 Museums From Around the World That You Can Visit Virtually. Travel+Leisure. https://www.travelandleisure.com/attractions/museumsgalleries/museums-with-virtual-tours

Sachs, M. E., Damasio, A., \& Habibi, A. (2015). The pleasures of sad music: A systematic review. Frontiers in Human Neuroscience, 9. https://doi.org/10.3389/fnhum.2015.00404

Samaroudi, M., Echavarria, K. R., \& Perry, L. (2020). Heritage in lockdown: Digital provision of memory institutions in the UK and US of America during the COVID-19 pandemic. Museum Management and Curatorship. https://www.tandfonline.com/doi/abs/10.1080/09647775.2020.1810483

Son, H., \& Lee, J. (2021). Does online shopping make people feel better? The therapeutic effect of online shopping on Korean female consumers' mood, self-esteem, and self-efficacy : 
Based on the context of fashion product shopping. Journal of Global Scholars of Marketing Science, O(0), 1-18. https://doi.org/10.1080/21639159.2020.1808821

Specker, E., Fekete, A., Trupp, M., \& Leder, H. (2021). Is a 'real' artwork better than a reproduction? A meta-analysis of the genuineness effect. Psychology of Aesthetics Creativity and the Arts. https://doi.org/10.1037/aca0000399

Specker, E., Forster, M., Brinkmann, H., Boddy, J., Pelowski, M., Rosenberg, R., \& Leder, H. (2020). The Vienna Art Interest and Art Knowledge Questionnaire (VAIAK): A unified and validated measure of art interest and art knowledge. Psychology of Aesthetics, Creativity, and the Arts, 14(2), 172-185. https://doi.org/10.1037/aca0000205

Staricoff, R., Loppert, S., Kirklin, D., \& Richardson, R. (2003). Integrating the arts into health care: Can we affect clinical outcomes. The Healing Environment: Without and Within. London: RCP, 63-79.

Tabachnick, B. G., \& Fidell, L. S. (2013). Using multivariate statistics (Vol. 6). Pearson Boston, MA.

Thomson, L. J., Lockyer, B., Camic, P. M., \& Chatterjee, H. J. (2018). Effects of a museumbased social prescription intervention on quantitative measures of psychological wellbeing in older adults. Perspectives in Public Health, 138(1), 28-38. https://doi.org/10.1177/1757913917737563

Tinkler, L., \& Hicks, S. (2011). Measuring Subjective Well-being. Office for National Statistics. Todd, C., Camic, P. M., Lockyer, B., Thomson, L. J., \& Chatterjee, H. J. (2017). Museum-based programs for socially isolated older adults: Understanding what works. Health \& Place, $48,47-55$. 
Tyack, C., Camic, P. M., Heron, M. J., \& Hulbert, S. (2017). Viewing art on a tablet computer: A well-being intervention for people with dementia and their caregivers. Journal of Applied Gerontology, 36(7), 864-894. https://doi.org/10.1177/0733464815617287

Tymoszuk, U., Perkins, R., Fancourt, D., \& Williamon, A. (2019). Cross-sectional and longitudinal associations between receptive arts engagement and loneliness among older adults. Social Psychiatry and Psychiatric Epidemiology, 1-10.

Tymoszuk, U., Perkins, R., Spiro, N., Williamon, A., \& Fancourt, D. (2020). Longitudinal associations between short-term, repeated, and sustained arts engagement and well-being outcomes in older adults. The Journals of Gerontology. Series B, Psychological Sciences and Social Sciences, 75(7), 1609-1619. https://doi.org/10.1093/geronb/gbz085

Ulrich, R., \& Gilpin, L. (2003). Healing Arts: Nutrition for the Soul. In Putting Patients First: Designing and Practicing Patient-Centered Care (pp. 117-146). Jossey Bass.

Unitt, C. (2020, May 7). Actually yes, people do want virtual museum tours. Cultural Digital Newsletter. https://www.chrisunitt.co.uk/2020/05/actually-yes-people-do-want-virtualmuseum-tours/

Wang, S., Mak, H. W., \& Fancourt, D. (2020). Arts, mental distress, mental health functioning \& life satisfaction: Fixed-effects analyses of a nationally-representative panel study. $B M C$ Public Health, 20(1), 208. https://doi.org/10.1186/s12889-019-8109-y

Wheatley, D., \& Bickerton, C. (2017). Subjective well-being and engagement in arts, culture and sport. Journal of Cultural Economics, 41(1), 23-45. https://doi.org/10.1007/s10824-0169270-0 
Wheatley, D., \& Bickerton, C. (2019). Measuring changes in subjective well-being from engagement in the arts, culture and sport. Journal of Cultural Economics, 43(3), 421442. https://doi.org/10.1007/s 10824-019-09342-7

White, M., Smith, A., Humphryes, K., Pahl, S., Snelling, D., \& Depledge, M. (2010). Blue space: The importance of water for preference, affect, and restorativeness ratings of natural and built scenes. Journal of Environmental Psychology, 30(4), 482-493. https://doi.org/10.1016/j.jenvp.2010.04.004

Zubala, A., Kennell, N., \& Hackett, S. (2021). Art therapy in the digital world: An integrative review of current practice and future directions. Frontiers in Psychology, 12. https://doi.org/10.3389/fpsyg.2021.600070 


\section{Footnotes}

${ }^{1}$ Interestingly, although one might surmise that a solution to loneliness requires being with other people or engaging at least in communal activity, one of the topics specifically addressed in Covid-19 times-related online art was loneliness, with several exhibitions featuring works on this theme (Jones, 2020; Knott, 2020).

${ }^{2}$ Note, we are not making any ontological claim regarding the definition of art, however we selected our stimuli so the painting was an example of objects highly popular and well-known to the general public as fine art, whereas the non-art condition had no clear connection to art and was focused on a different aspect of culture, namely the cultural/history of food. 


\section{Tables and Figures}

\section{Table 1}

Average participant self-report ratings for each time condition per group (means and standard deviations)

\begin{tabular}{llllllllll}
\hline \multicolumn{1}{c}{ Variable } & & \multicolumn{4}{c}{ Water-lilies $^{\mathrm{a}}$} & \multicolumn{5}{c}{ Bento $^{\mathrm{b}}$} \\
\cline { 2 - 11 } & \multicolumn{3}{c}{ Pre } & \multicolumn{2}{c}{ Post } & \multicolumn{2}{c}{ Pre } & \multicolumn{2}{c}{ Post } \\
\hline Negative mood & $M(S D)$ & 3.08 & $(1.32)$ & 2.75 & $(1.36)$ & 2.95 & $(1.53)$ & 2.64 & $(1.60)$ \\
Positive mood & $M(S D)$ & 4.31 & $(1.39)$ & 4.75 & $(1.18)$ & 4.69 & $(1.24)$ & 4.86 & $(1.34)$ \\
Anxiety & $M(S D)$ & 3.60 & $(1.18)$ & 3.19 & $(1.16)$ & 3.31 & $(1.32)$ & 3.09 & $(1.24)$ \\
Loneliness & $M(S D)$ & 3.49 & $(1.10)$ & 3.32 & $(0.93)$ & 3.50 & 0.93() & 3.34 & $(0.97)$ \\
Life satisfaction & $M(S D)$ & 4.54 & $(1.28)$ & 4.95 & $(1.30)$ & 4.52 & $(1.21)$ & 4.41 & $(1.40)$ \\
wellbeing & $M(S D)$ & 4.26 & $(1.12)$ & 4.49 & $(1.11)$ & 4.41 & $(1.14)$ & 4.66 & $(1.14)$ \\
\hline
\end{tabular}

Note. $N^{\mathrm{a}}=36, N^{\mathrm{b}}=42$, (see exclusion criteria). For a positive impact to occur, negative mood, anxiety, and anxiety loneliness should decrease whereas positive mood, satisfaction with life and wellbeing should increase. 
Table 2

Stepdown analysis statistics

\begin{tabular}{|c|c|c|c|c|c|c|c|c|c|c|}
\hline Variable & Analysis & Covariate & Pre & Post & $d f$ & $F$ & $p(a d j)$ & $\eta_{p}^{2}$ & \multicolumn{2}{|c|}{$90 \% \mathrm{CI}$} \\
\hline & & & $M(S D)$ & $M(S D)$ & & & & & LL & UL \\
\hline $\begin{array}{l}\text { Negative } \\
\text { mood }\end{array}$ & Univariate & - & $3.01(1.43)$ & $2.69(1.49)$ & 1,77 & 7.81 & .039 & 0.09 & 0.15 & 0.21 \\
\hline \multirow{3}{*}{$\begin{array}{l}\text { Positive } \\
\text { mood }\end{array}$} & Univariate & - & $4.51(1.32)$ & $4.81(1.26)$ & 1,77 & 5.92 & .104 & 0.07 & 0 & 0.18 \\
\hline & Step-down & $\begin{array}{l}\text { Negative } \\
\text { mood }\end{array}$ & & & 1,76 & 49.72 & $<.001$ & 0.40 & 0.25 & 0.51 \\
\hline & Step-down & & & & 1,76 & 0.83 & .353 & 0.01 & 0 & 0.08 \\
\hline \multirow[t]{4}{*}{ Anxiety } & Univariate & - & $3.44(1.26)$ & $3.14(1.20)$ & 1,77 & 5.92 & .01 & 0.12 & 0.03 & 0.24 \\
\hline & Step-down & $\begin{array}{l}\text { Negative } \\
\text { mood }\end{array}$ & & & 1,75 & 29.71 & $<.001$ & 0.28 & 0.15 & 0.41 \\
\hline & Step-down & $\begin{array}{l}\text { Positive } \\
\text { mood }\end{array}$ & & & 1,75 & 13.72 & $<.001$ & 0.15 & 0.05 & 0.28 \\
\hline & Step-down & & & & 1,75 & 3.68 & .406 & 0.05 & 0 & 0.15 \\
\hline \multirow[t]{5}{*}{ Loneliness } & Univariate & - & $3.50(1.00)$ & $3.33(0.95)$ & 1,77 & 9.64 & .016 & 0.11 & 0.03 & 0.23 \\
\hline & Step-down & $\begin{array}{l}\text { Negative } \\
\text { mood }^{\text {a }}\end{array}$ & & & 1,74 & 3.47 & .067 & 0.06 & 0 & 0.16 \\
\hline & Step-down & $\begin{array}{l}\text { Positive } \\
\text { mood }\end{array}$ & & & 1,74 & 1.26 & .265 & 0.02 & 0 & 0.09 \\
\hline & Step-down & Anxiety & & & 1,74 & 6.14 & .016 & 0.08 & 0 & 0.18 \\
\hline & Step-down & & & & 1,74 & 4.54 & .219 & 0.05 & 0 & 0.14 \\
\hline \multirow{6}{*}{$\begin{array}{l}\text { Life } \\
\text { satisfaction }\end{array}$} & Univariate & - & $4.53(1.24)$ & $4.50(1.35)$ & 1,77 & 0.00 & $>.999$ & 0.01 & 0 & 0.01 \\
\hline & Step-down & $\begin{array}{l}\text { Negative } \\
\text { mood }\end{array}$ & & & 1,73 & 0.01 & .932 & 0.01 & 0 & 0.7 \\
\hline & Step-down & $\begin{array}{l}\text { Positive } \\
\text { mood }\end{array}$ & & & 1,73 & 0.18 & 281 & 0.01 & 0 & 0.09 \\
\hline & Step-down & Anxiety & & & 1,73 & 0.14 & .705 & 0.00 & 0 & 0.01 \\
\hline & Step-down & Loneliness & & & 1,73 & 0.13 & .721 & 0.00 & 0 & 0.05 \\
\hline & Step-down & & & & 1,73 & 0.53 & .353 & 0.00 & 0 & 0.05 \\
\hline
\end{tabular}




\begin{tabular}{|c|c|c|c|c|c|c|c|c|c|c|}
\hline Variable & Analysis & Covariate & Pre & Post & $d f$ & $F$ & $p(a d j)$ & $\eta_{p}^{2}$ & $90 \% \mathrm{C}$ & \\
\hline \multirow[t]{7}{*}{ Wellbeing } & & & $M(S D)$ & $M(S D)$ & & & & & LL & UL \\
\hline & Step-down & $\begin{array}{l}\text { Negative } \\
\text { mood }\end{array}$ & $4.34(1.12)$ & $4.58(1.12)$ & 1,72 & 6.19 & .015 & 0.08 & 0.01 & 0.19 \\
\hline & Step-down & $\begin{array}{l}\text { Positive } \\
\text { mood }\end{array}$ & & & 1,72 & 4.57 & .036 & 0.06 & 0 & 1.16 \\
\hline & Step-down & Anxiety & & & 1,72 & 1.31 & .256 & 0.00 & 0 & 0.06 \\
\hline & Step-down & Loneliness & & & 1,72 & 3.26 & .075 & 0.04 & 0 & 0.15 \\
\hline & Step-down & $\begin{array}{l}\text { Life } \\
\text { satisfaction }\end{array}$ & & & 1,72 & 0.30 & .588 & 0.02 & 0 & 0.10 \\
\hline & Step-down & & & & 1,72 & 3.44 & .353 & 0.04 & 0 & 0.14 \\
\hline
\end{tabular}

Note. $\mathrm{CI}=$ confidence interval; $\mathrm{LL}=$ lower limit; $\mathrm{UL}=$ upper limit. ${ }^{\text {a }}$ Before exclusion of three multivariate outliers, negative mood explained a significant proportion of the change in loneliness, $\mathrm{p}=.032$. 


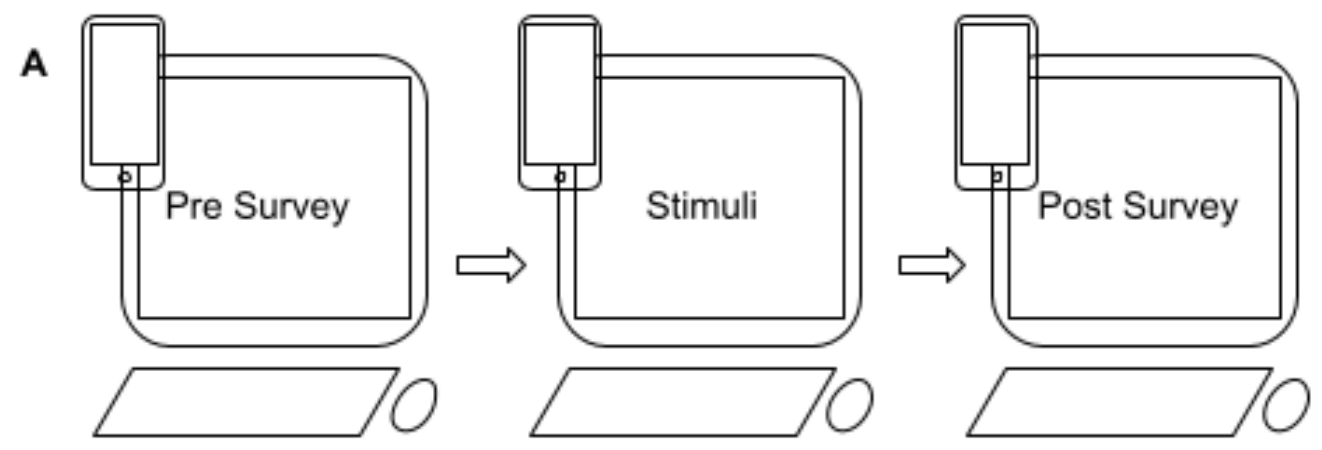

B Water-lilies Main Image

Bento Main Image
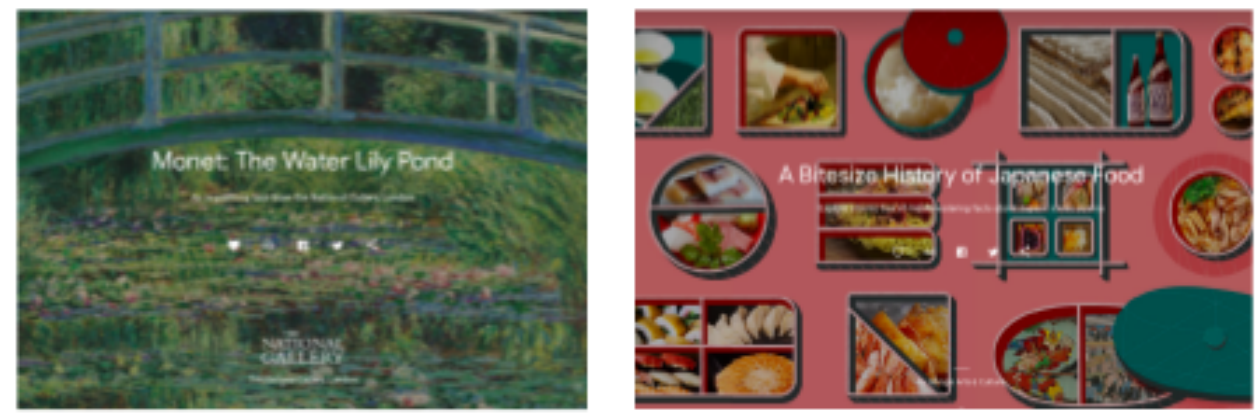

Figure 1. Study design, stimuli composition. a, the procedure was a pre-post design which could be taking on both personal computers and smartphones. b, stimuli from Google Arts and Culture were interactive, where participants could scroll through smaller compositions of one main image, shown above. 
a.

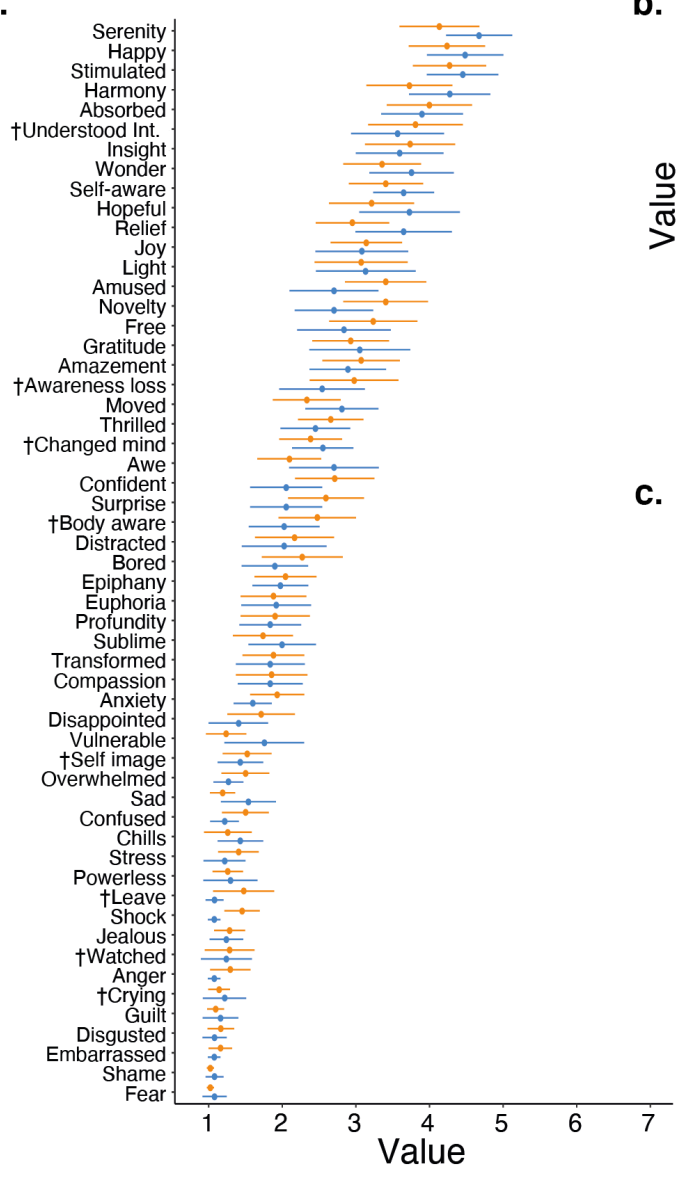

b.

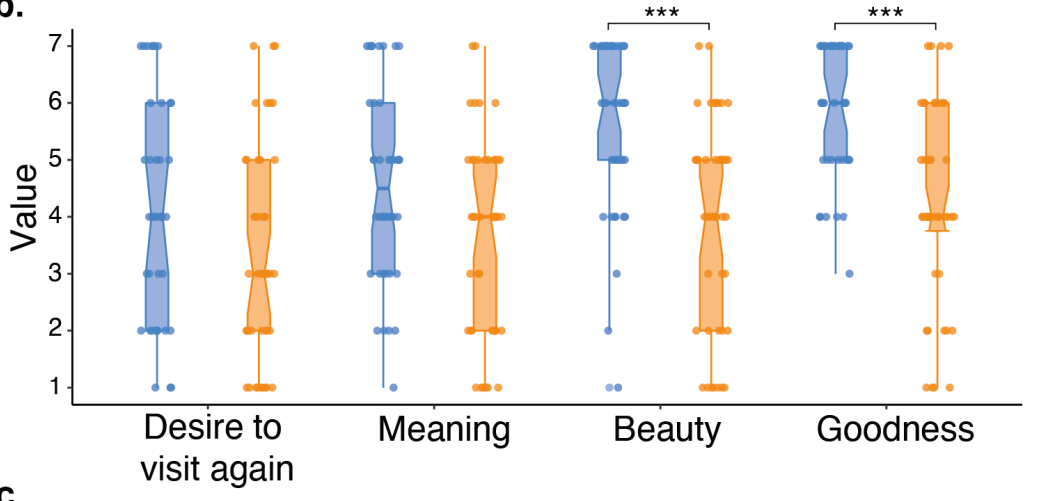

Condition $\square$ Water-lilies $\square$ Bento

Figure 2. a shows means and $95 \% \mathrm{CI}$ intervals for participants ratings of cognitive-emotional states during the online cultural engagements for the Water-lilies (light blue) and Bento (light orange) conditions. ${ }^{+}$shorthand labels, see Supplementary for the complete labels. b box plots showing the ratings for aesthetic evaluation for the Water-lilies and Bento conditions, showing that in general Water-lilies condition was rated more highly. $\mathbf{c}$ pie chart showing percentages of participants who reported to see art or something else while engaging online with the Water-lilies and Bento conditions. 
a.

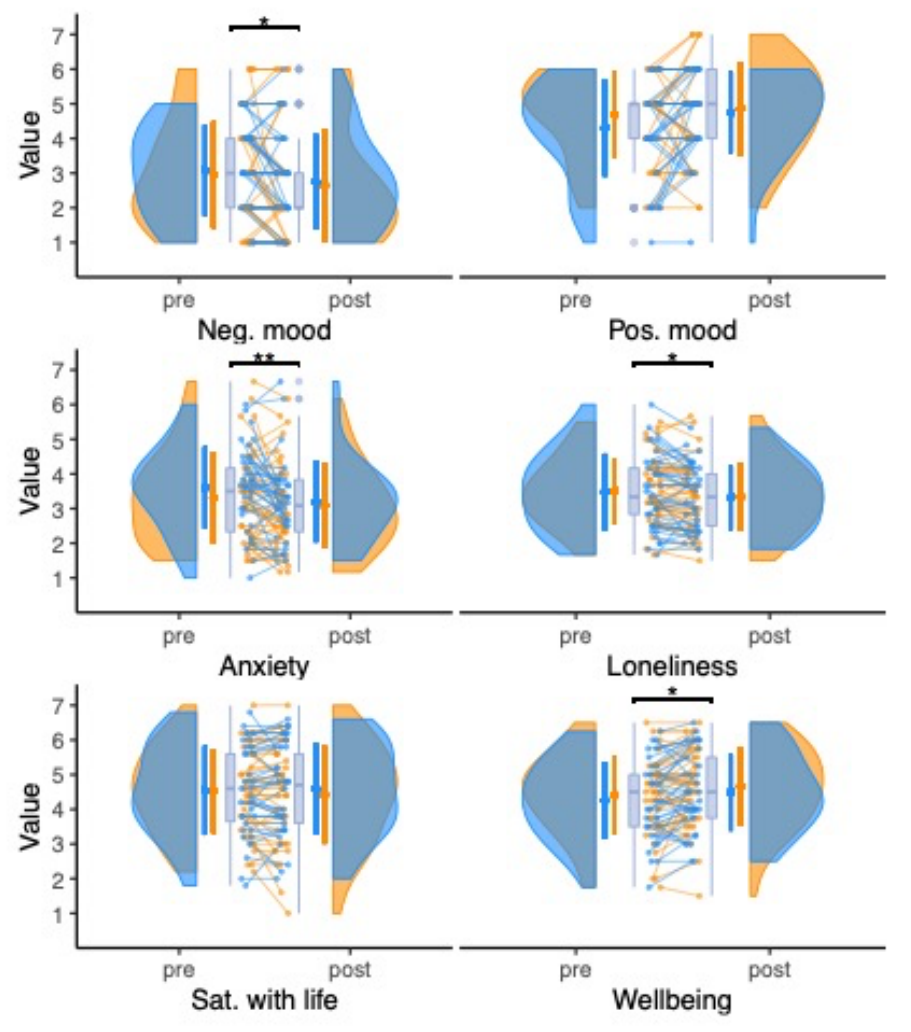

b.

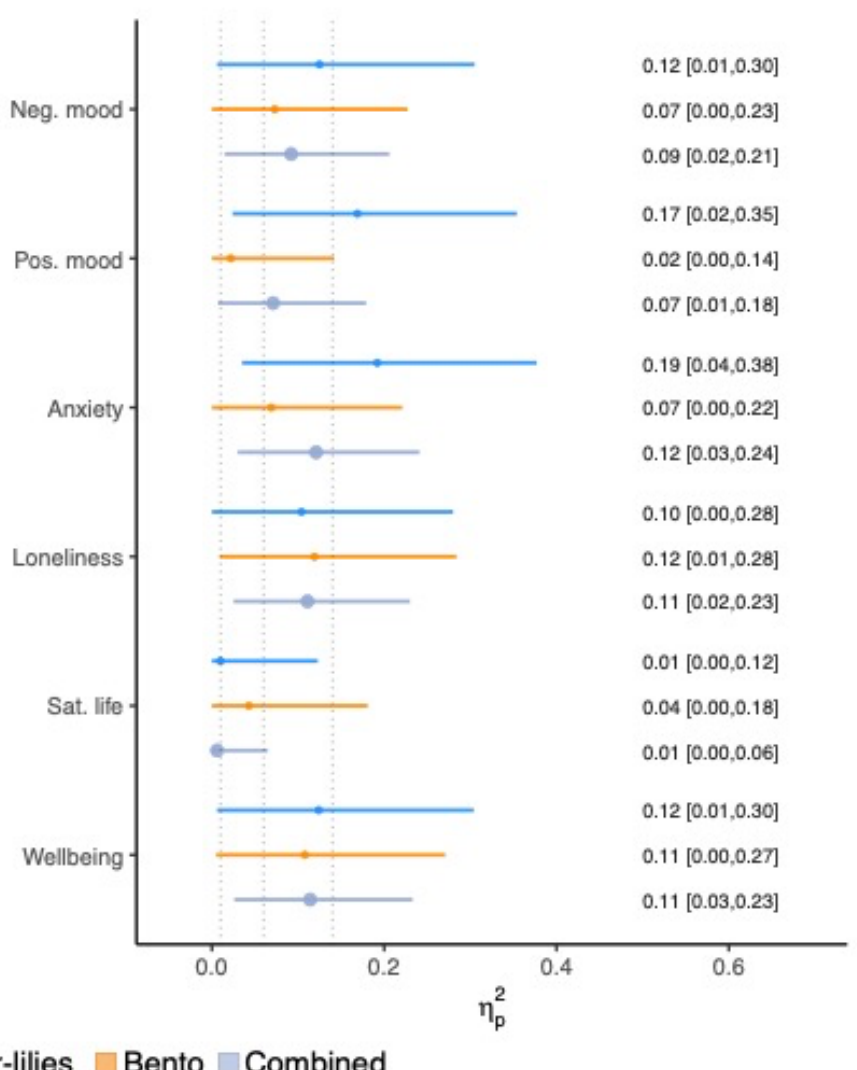

Figure 3. a Half violin plots showing the distributions of rating pre and post the online cultural experience. Vertical bars show the 95\% CI and means; Box plots show SD and Medians for the combined samples; Dots represents individual ratings; Slopes represent changes between pre and post. Images were computed adapting van Langen's tutorial (Allen et al., 2021; see https://github.com/jorvlan/open-visualizations); b Forest plot of the effect sizes (partial eta square) of the online experience on the six DVs. Vertical dashed lines are used to indicate intervals usually used to interpret the strength of the effect. 

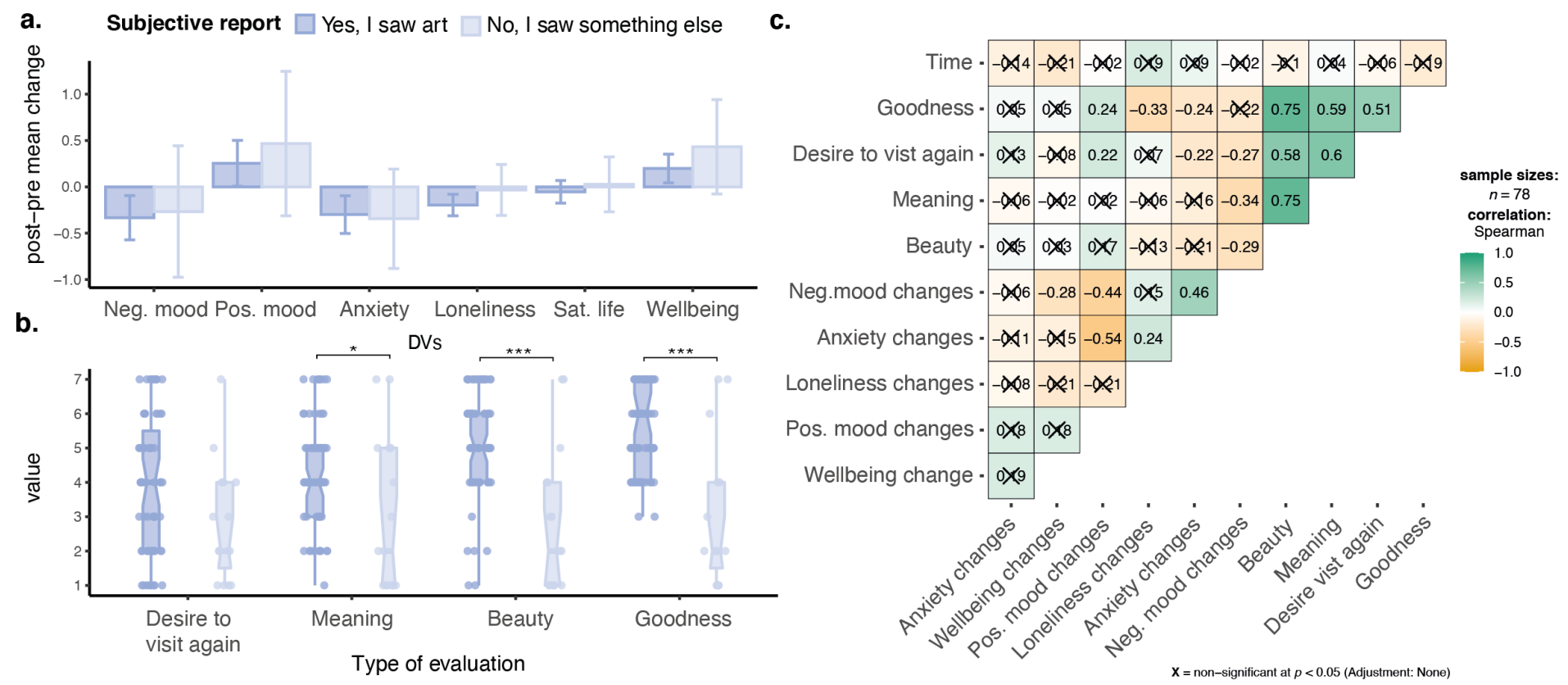

Figure 4. Subjective experience of participants, including a DV changes grouped by perception of if participants considered they say art or not, bar plots showing the breakdown of changes in Wellbeing DVs. Error bars represent 95\% CI. b. box plots showing the breakdown of the aesthetic ratings grouped by subjective reports (i.e., seeing art), Error bars represent SD. * p $<.05, * * * \mathrm{p}<.001 . \mathrm{p}$ values uncorrected. $\mathbf{b}$ appraisals shown broken down by I saw art or not. $\mathbf{c}$ correlations between viewing time, appraisals and changes in Wellbeing DVs. Correlation plot was computed using ggstatplot (Patil, 2021). 